\title{
Identification of a New Potent Inhibitor Targeting KRAS in Non-small Cell Lung Cancer Cells
}

\author{
Chun Xie ${ }^{1 \dagger}$, Ying Lit ${ }^{1+}$, Lan-Lan Li ${ }^{2}$, Xing-Xing Fan', Yu-Wei Wang ${ }^{1}$, Chun-Li Wei ${ }^{1}$, \\ Liang Liu ${ }^{1 *}$, Elaine Lai-Han Leung ${ }^{1 *}$ and Xiao-Jun Yao ${ }^{1,2 *}$
}

\begin{abstract}
'State Key Laboratory of Quality Research in Chinese Medicine, Macau Institute for Applied Research in Medicine and Health, Macau University of Science and Technology, Macau, China, ${ }^{2}$ State Key Laboratory of Applied Organic Chemistry and Department of Chemistry, Lanzhou University, Lanzhou, China
\end{abstract}

\section{OPEN ACCESS}

Edited by:

Leonardo G. Ferreira,

University of São Paulo, Brazil

Reviewed by:

Andrea llari,

Istituto di Biologia e Patologia

Molecolari (CNR), Italy

Chakrabhavi Dhananjaya Mohan,

University of Mysore, India

*Correspondence:

Liang Liu

Iliu@must.edu.mo

Elaine Lai-Han Leung

Ihleung@must.edu.mo

Xiao-Jun Yao

xjyao@must.edu.mo

tThese authors have contributed equally to this work.

Specialty section:

This article was submitted to Experimental Pharmacology and Drug

Discovery,

a section of the journal

Frontiers in Pharmacology

Received: 06 July 2017 Accepted: 30 October 2017 Published: 14 November 2017

Citation:

Xie C, Li Y, Li L-L, Fan X-X,

Wang $Y-W$, Wei C-L, Liu L,

Leung EL-H and Yao X-J (2017)

Identification of a New Potent Inhibitor

Targeting KRAS in Non-small Cell

Lung Cancer Cells.

Front. Pharmacol. 8:823.

doi: 10.3389/fphar.2017.00823
KRAS (v-Ki-ras2 Kirsten rat sarcoma viral oncogene homolog) is an oncogenic driver with mutations in $30 \%$ of non-small cell lung cancer (NSCLC). However, there is no effective clinical drug even though it has been identified as an oncogene for 30 years. In this study, we identified a small molecule inhibitor compound 0375-0604 targeting KRAS by using molecular docking based virtual screening approach. Compound 03750604 had a good binding affinity to KRAS in vitro and exhibited cytotoxicity in oncogenic KRAS expressing NSCLC cell lines. Further mechanism study showed that compound 0375-0604 can block the formation of the complex of guanosine triphosphate (GTP) and KRAS in vitro. In addition, compound 0375-0604 inhibited KRAS downstream signaling pathway RAF/MEK/ERK and RAF/PI3K/AKT. Finally, we also found that this compound can inhibit the cell growth through G2/M cell cycle arrest and induce apoptosis on the NSCLC cell lines harboring KRAS mutation. Therefore, compound 0375-0604 may be considered as a potential KRAS inhibitor for treatment of NSCLC carrying KRAS oncogene.

Keywords: KRAS, NSCLC, small molecule inhibitor, molecular docking

\section{INTRODUCTION}

In lung cancer, NSCLC is the majority category and accounts for 85\% (Ettinger et al., 2017). The overall survival of patients with advanced or metastatic NSCLC is still dismal (Shima et al., 2015; Lazo and Sharlow, 2016). With the development of modern sequencing technology, NSCLC was further classified into different subtypes according to the frequency of gene mutation, such as EGFR, ALK, MET, ROS-1, KRAS (Chen et al., 2014). Mutated KRAS genes are frequently found in human cancers, especially in approximately $30 \%$ of lung cancer. Ninety seven percent of mutated KRAS occurs in exon 2 and 3, including amino acid G12, G13, and Q61 (Karachaliou et al., 2013). Due to high morbidity and mortality, a great deal of attention has been paid to study NSCLC with KRAS mutations. However, there is still no direct and effective drug for clinical use (Jancik et al., 2010; Gysin et al., 2011; Vasan et al., 2014; Papke and Der, 2017).

KRAS plays an important role in normal cell development, such as proliferation and differentiation (Pylayeva-Gupta et al., 2011; Santarpia et al., 2012). As a small GTPase, KRAS normally cycles between inactive GDP-bound state and active GTP-bound state, which are tightly regulated by GTPase-activating proteins (GAPs) and Guanine nucleotide exchange factors (GEFs), respectively (Maurer et al., 2012; Burns et al., 2014; Leshchiner et al., 2015). However, mutant 
KRAS impairs its GAPs activity, which locks KRAS at the active state (Smith et al., 2013; Clausen et al., 2015). Thereby mutant KRAS promotes its interaction with a variety of effector proteins and activates downstream signaling events, and finally results in tumor formation (Bos et al., 2007; Zimmermann et al., 2013; Lito et al., 2016). Therefore, it is urgently needed to find effective inhibitors to target and inhibit oncogenic KRAS in cancers.

To date, there are mainly three strategies for the discovery of potent KRAS inhibitors: (1) to inhibit KRAS membrane targeting (Laheru et al., 2012; Prakash and Gorfe, 2013; Chavan et al., 2015; Cox et al., 2015); (2) to directly target KRAS (Wang et al., 2012; Cromm et al., 2015; Leshchiner et al., 2015; Brock et al., 2016; Trinh et al., 2016); (3) to inhibit interaction between KRAS and its downstream effectors (Athuluri-Divakar et al., 2016; Upadhyaya et al., 2016; Keeton et al., 2017). However, there are multiple escape pathways by process of posttranslation for inhibiting KRAS membrane targeting (Rowinsky et al., 1999; Van Cutsem et al., 2004). Inhibitor lonafarnib and tipifarnib showed effective inhibition to KRAS mutations through blocking prenylation of RAS, but failed in clinical trial, as the geranylgeranylation could be in replacement of prenylation when the farnesyltransferase was inhibited by these two inhibitors (Berndt et al., 2011). Additionally, it may be not a good choice to inhibit interaction between KRAS and its downstream effectors for developing KRAS inhibitors. Firstly, there are a lot of downstream effector proteins of KRAS involving in multiple signaling pathways, such as RAF (MAP kinase pathway), PI3K (AKT/mTOR pathway), and RalGDS (Ral pathway). Secondly, these effectors are not only highly complex but also regulating multiple pathways (Downward, 2003). Arguably, designing a small molecule inhibitor directly targeting KRAS may be one of the most effective ways. However, the biggest challenges to develop direct KRAS inhibitor are the high binding affinity between KRAS and GDP/GTP in the picomolar range and the relatively flat surface without deep hydrophobic pockets in KRAS protein (Ledford, 2015; Vo et al., 2016). Notably, in recent years, several reported works have shown the novel transient pockets on KRAS protein surfaces, which recover the hope in the development of KRAS inhibitors (Prakash and Gorfe, 2013; Wang et al., 2014).

In this study, we aimed to identify effective and potential KRAS inhibitors by directly targeting KRAS to prevent cell growth of NSCLC harboring KRAS mutation. We performed a molecular docking-based virtual screening from a small molecule database to screen KRAS inhibitors. A potential inhibitor compound 0375-0604 was found to bind to KRAS and exhibit the effective cytotoxicity to KRAS mutant NSCLC cell lines.

\section{RESULTS}

\section{The Binding Mode between Compound 0375-0604 and KRAS}

To discover potential small molecules targeting KRAS, virtual screening based on molecular docking was performed on Chemdiv library with about 1.36 million compounds. The most promising compound 0375-0604 (Figure 1A) was selected for further study. It was shown that the benzothiazole ring of 0375-0604 inserted into the binding pocket of KRAS with the linker sulfur atom exposed to solvent environment (Figures 1B,C). The amino group of compound 0375-0604 formed H-bond interactions with the backbone of Met67 and the side chain of Glu37, locating in switch I and II regions of KRAS $^{\mathrm{G} 12 \mathrm{D}}$, respectively. At the same time, 0375-0604 formed polar contacts and hydrophobic contacts with the surrounding residues. 0375-0604 bound to KRAS ${ }^{\mathrm{G} 12 \mathrm{C}}$ with a similar manner (Figure 1D) as $\mathrm{KRAS}^{\mathrm{G} 12 \mathrm{D}}$, except for the orientation of chlorobenzene rings. The orientation of benzothiazole ring and chlorobenzene rings of 0375-0604 switched in KRASQ61H (Figure 1E). Docking score of 0375-0604 in various systems was shown in Figure 1F.

\section{The Binding Affinity of Compound 0375-0604 with KRAS}

To determine the binding affinity of this small molecule with KRAS, we used biolayer interferometry assay (BLI) (Rich and Myszka, 2007), a label-free technology, to measure biomolecular interactions. Different concentration of compound 0375-0604 was measured in real time by association with both-labeled KRAS protein, which was immobilized on the streptavidin (SA) biosensors. All the association/dissociation binding curves was shown in Figure 2A, and we further performed the steady-state analysis (Figure 2B) with ForteiBio data analysis software to obtain the binding affinity with $\mathrm{K}_{\mathrm{D}}$ value of $92 \mu \mathrm{M}$ (Figure 2C), which demonstrated their direct and reversible interaction with KRAS.

\section{Compound 0375-0604 Decreased Cell Viability of NSCLC Cells with KRAS Mutations}

Since compound 0375-0604 bound to KRAS in vitro, we further determined its cytotoxicity in NSCLC cell lines harboring mutant KRAS by using MTT assay, including H2122 (KRAS ${ }^{\mathrm{G} 12 \mathrm{C}}$ ), $\mathrm{H} 358$ (KRAS $^{\mathrm{G} 12 \mathrm{C}}$ ) and H460 (KRAS ${ }^{\mathrm{Q} 61 \mathrm{H}}$ ) cell lines. Cells were incubated with a range of compound 0375-0604 concentrations $(0,25,50,100 \mu \mathrm{M})$ for 24, 48, and $72 \mathrm{~h}$. As shown in Figure 3, compound 0375-0604 inhibited three NSCLC cell lines in a doseand time-dependent manner, but not in normal lung fibroblast cell line CCD-19Lu. Importantly, we found that the $\mathrm{IC}_{50}$ value of compound 0375-0604 in H2122, H358 and H460 cells were up to 6-fold less than that of CCD-19Lu cells, which suggested that compound 0375-0604 showed strong inhibition selectivity in NSCLC cells.

\section{Compound 0375-0604 Blocked GTP-KRAS Formation in NSCLC Cells}

Actually, mutant KRAS would interfere the balance between GEFs and GAPs, resulting in locking in the active GTP-bound KRAS state and aberrant stimulation of its downstream signaling. Hence, KRAS inhibitors should reduce the formation of GTPKRAS to disrupt the mutant KRAS function.

In order to know whether compound 0375-0604 could inhibit activation of KRAS, we performed RAS activation assay to 


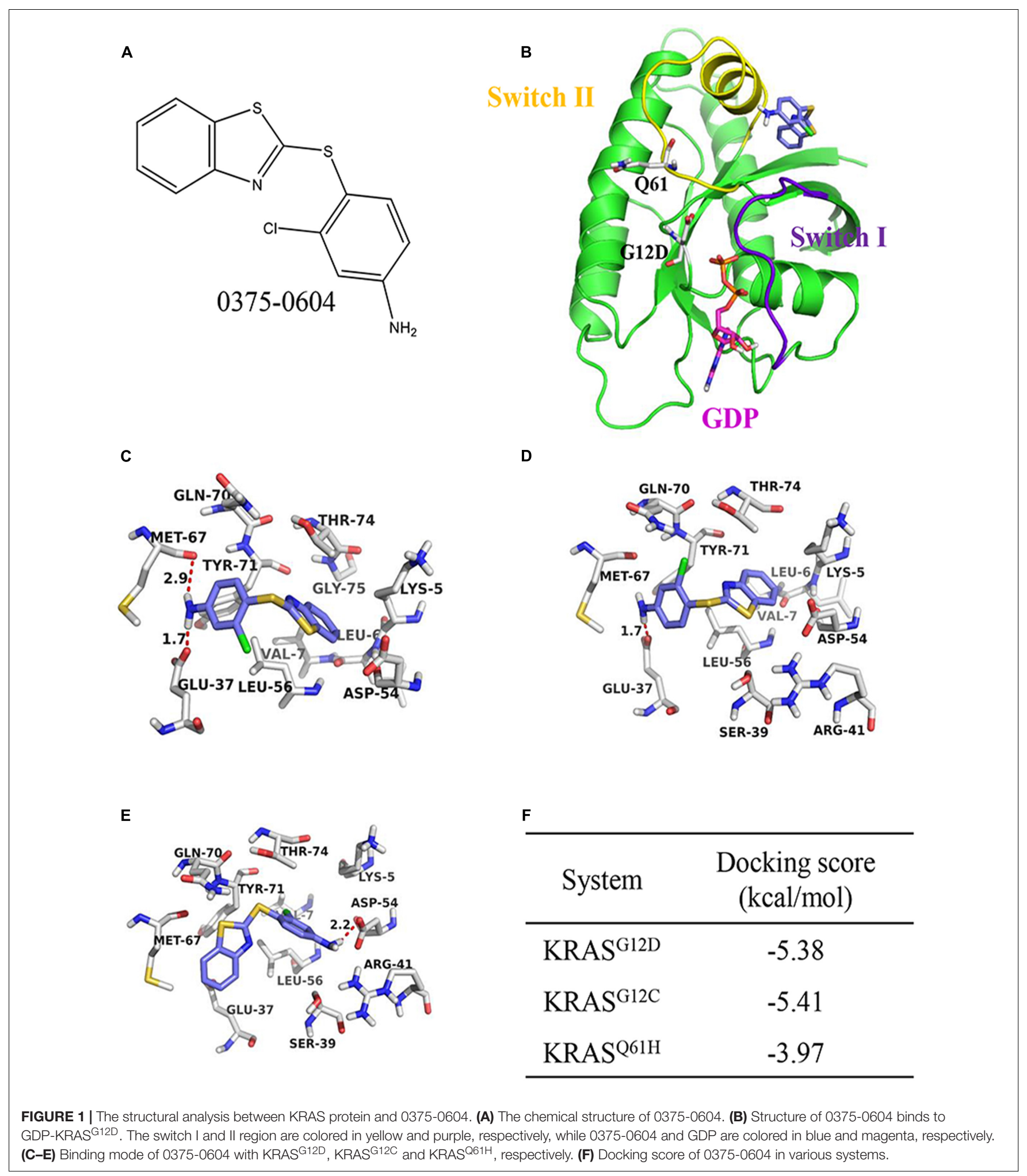

examine the formation of GTP-bound KRAS after treatment with a range of concentrations of compound 0375-0604 in $\mathrm{H} 2122$, $\mathrm{H} 358$ and $\mathrm{H} 460$ cells at $24 \mathrm{~h}$. As shown in Figure 4, the formation of GTP-KRAS was inhibited in KRAS mutant NSCLC by compound 0375-0604 treatment, compared to total amount of KRAS, suggesting this small molecule could partially rescue this unbalance resulted from mutant KRAS. 

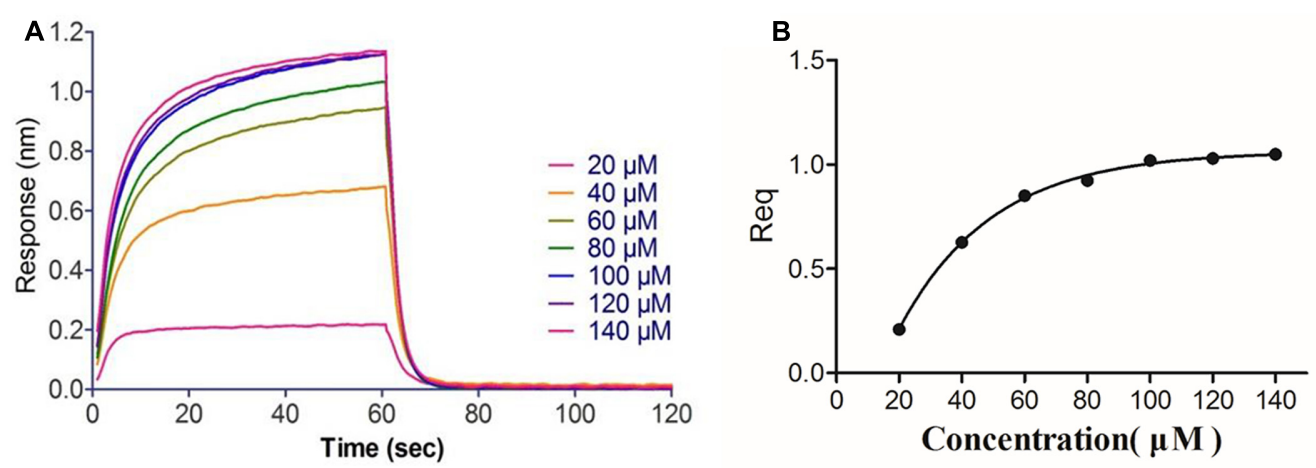

C

Kinetics of the interaction between KRAS and 0375-0604 by BLI

\begin{tabular}{ccc}
\hline $\mathrm{K}_{\mathrm{D}}(\mu \mathrm{M})$ & $\mathrm{k}_{\text {on }}(1 / \mathrm{Ms})$ & $\mathrm{k}_{\mathrm{dis}}(1 / \mathrm{s})$ \\
\hline 92 & $2.9 \times 10^{+03}$ & $2.6 \times 10^{-01}$ \\
\hline
\end{tabular}

FIGURE 2 | The binding affinity of compound 0375-0604 with KRAS was determined by interferometry studies. (A) Binding curves of varying concentrations of compound 0375-0604 to the immobilized KRAS protein. (B) Steady-state analysis of the binding curves. (C) The binding affinity (KD) of KRAS for compound 0375-0604 was determined. Experimental data for association and dissociation are represented as shown.

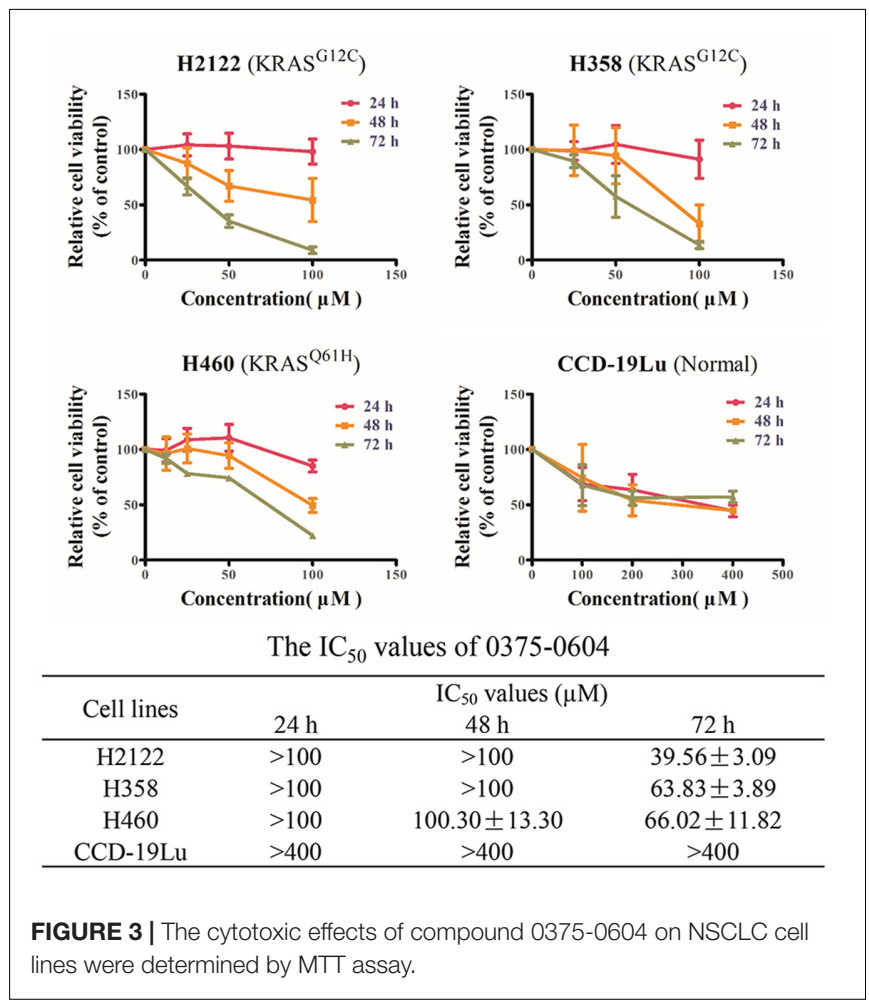

\section{Compound 0375-0604 Inhibited the Activation of KRAS Downstream Signaling Pathway}

Active KRAS stimulates downstream signaling pathways, especially for RAF/MEK/ERK and RAF/PI3K/AKT pathway, and

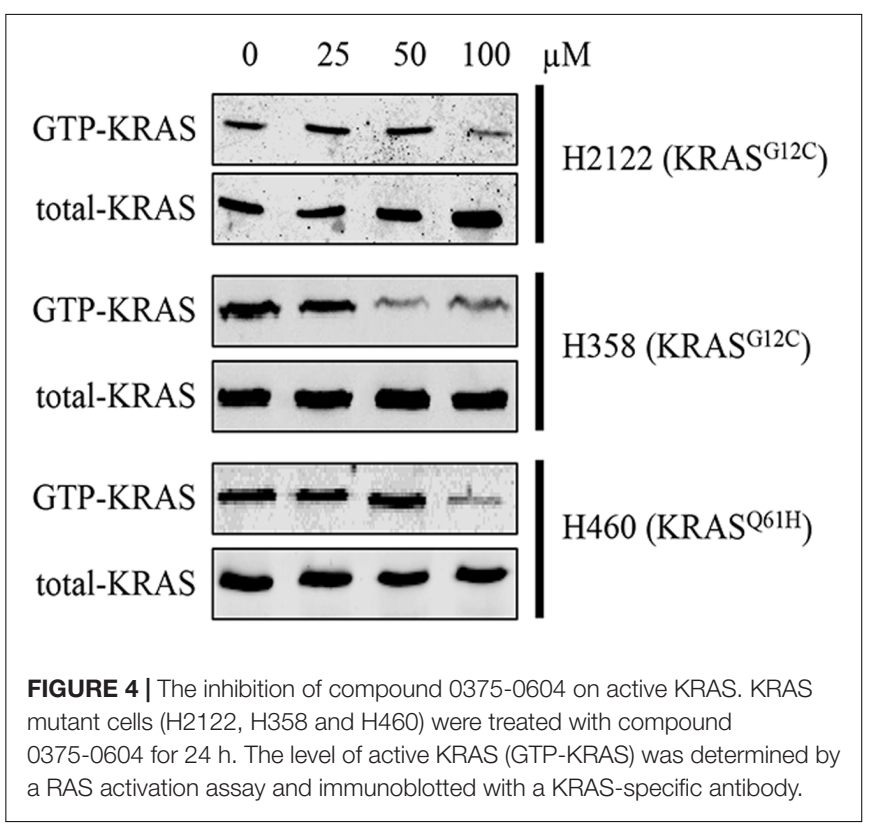

then induces cell proliferation. Therefore, to investigate the effect of compound 0375-0604, we examined the phosphorylation levels of CRAF, AKT and ERK in NSCLC cell lines to monitor the impact of KRAS signaling by treatment with this compound for $48 \mathrm{~h}$. As expected, compound 0375-0604 reduced the levels of phosphorylation of CRAF and AKT in a dose-dependent manner in all three NSCLC cell lines (Figure 5), which indicated that compound 0375-0604 may block oncogenic KRAS function through inhibiting its downstream signaling pathways. 


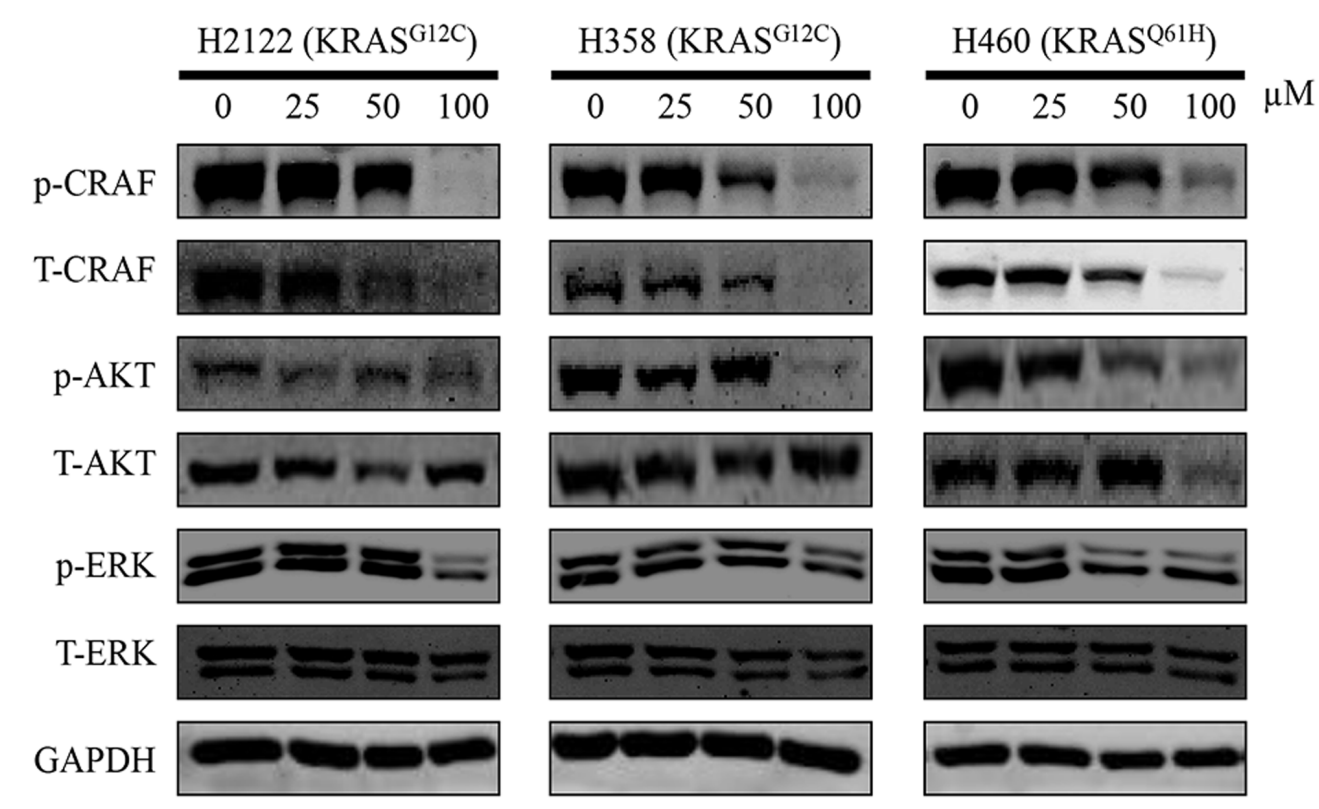

FIGURE 5 | The inhibitive effect of compound 0375-0604 on the KRAS signaling pathways in different NSCLC cell lines. Three NCSLC cell lines were treated with compound 0375-0604 for $48 \mathrm{~h}$. The levels of phospho(p)-CRAF, p-ERK, p-AKT, CRAF, AKT, ERK and GAPDH were determined by western blot analysis. Untreated cells were used as a control. A representative of at least three independent experiments for each cell line is showed.

\section{Compound 0375-0604 Induced Cell Cycle Arrest and Apoptosis in NSCLC}

Since compound 0375-0604 significantly inhibited cell viability of NSCLC cells with KRAS mutation, we examined whether compound 0375-0604 exhibited cytotoxicity by cell cycle arrest or apoptotic effect in H2122, H358 and H460 cells. Cells were treated with the indicated concentrations of compound 0375-0604 for 24, 48, and $72 \mathrm{~h}$. Flow cytometric analysis showed that after $24 \mathrm{~h}$ treatment, the percentage of cells significantly decreased in G0/G1 phase while remarkably increased in G2/M phase (Figure 6A). In addition, compound 0375-0604 induced a significantly increased apoptosis for $48 \mathrm{~h}$ in NSCLC cell lines (Figure 6B). These result suggested that compound 0375-0604 may block cell proliferation and cause cell death via induction of G2/M cell cycle arrest or/and apoptosis in H2122, H358, and H460 cells.

\section{DISCUSSION}

In this study, we identified and characterized a small-molecule compound 0375-0604 as a new KRAS inhibitor. By using molecular docking approach, we found that compound 03750604 bound to the switch regions (switch I and II) of KRAS. KRAS conformation changes and its downstream signals are activated when its switch regions, either switch I or switch II, interact with GTP. A remarkable feature of compound 0375-0604 is that it formed two hydrogen bonds interaction with the backbone of Met67 and the side chain of Glu37, which are located in switch I and switch II, respectively. These two key hydrogen bonds could partially stabilize the interaction of KRAS and
0375-0604. The docking calculation indicated compound 03750604 exhibited potent binding affinity with KRAS. In addition, the chemical structure of this inhibitor has more potential to be modified and achieve more potent and effective inhibition to oncogenic KRAS NSCLC. Based on the docking result, the binding affinity of compound 0375-0604 with KRAS protein was further determined by using BLI and $K_{D}$ value was $92 \mu \mathrm{M}$, which suggested that $0375-0604$ could bind to KRAS with good performance.

There are two most extensively RAS-mediated pathways: PI3K/AKT/mTOR and RAF/MEK/ERK pathway (Papke and Der, 2017). The PI3K/AKT/mTOR pathway represents an important intracellular signaling pathway, which involved in transition of cell cycle. It is directly related to cell proliferation, cancer progress and longevity (Bryant et al., 2014). The RAF/MEK/ERK pathway is a chain of proteins in cell that communicates a signal from a receptor on the surface of cell to the DNA in the nucleus of cell. The RAF serine/threonine kinases (ARAF, BRAF and CRAF) are arguably the most important effectors of mutant RAS-dependent cancer growth, as they have a key driver role in RAS-mediated oncogenesis. In our study, we found that compound 0375-0604 could reduce the activation levels of AKT, CRAF and ERK and block the activation of KRAS downstream signaling pathways in NSCLC.

KRAS binds to GTP in its active state and then influences the expression of downstream genes involved in crucial pathways on regulating cell growth, differentiation and apoptosis ( $\mathrm{Lu}$ et al., 2016). Compound 0375-0604 showed a strong anti-cancer activity by inhibiting the activation of KRAS proteins, and caused G2/M cell cycle arrest at the early stage and induced apoptosis 

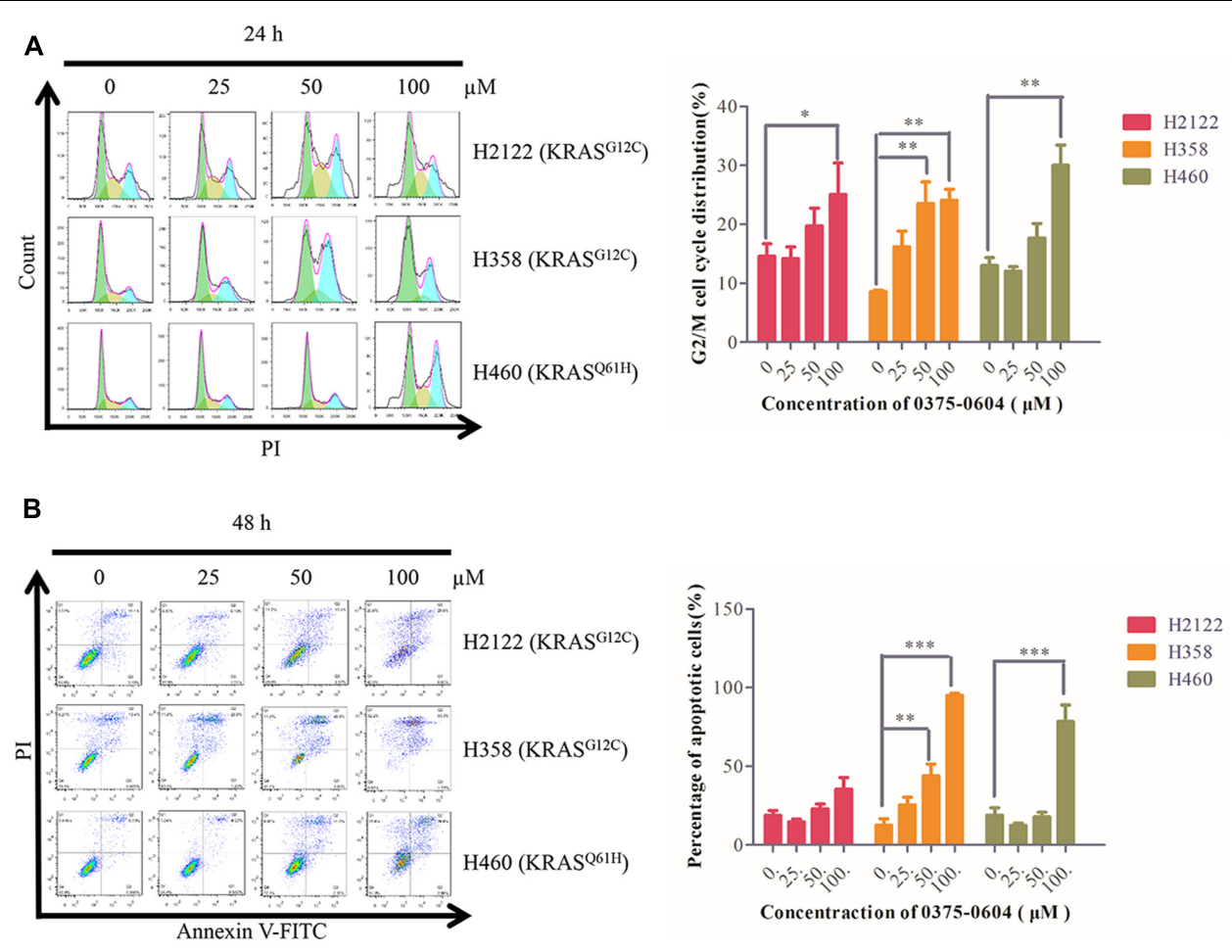

FIGURE 6 | Cell cycle arrest and apoptosis were induced by compound 0375-0604 in three NSCLC cell lines (H2122, H358 and H460). (A) Flow cytometric analysis of cell cycle arrest with compound 0375-0604 at different concentrations $(0,25,50,100 \mu \mathrm{M})$ was examined for $24 \mathrm{~h}$. (B) Flow cytometric analysis of cell apoptosis with different concentrations $(0,25,50,100 \mu \mathrm{M})$ of compound $0375-0604$ for $48 \mathrm{~h}$ was determined.

at the later stage in $\mathrm{H} 2122, \mathrm{H} 358$ and $\mathrm{H} 460$ cell lines harboring KRAS oncogene.

In summary, we identified a new small molecule compound 0375-0604 that bound to KRAS ${ }^{\mathrm{G} 12 \mathrm{D}}$, KRAS ${ }^{\mathrm{G} 12 \mathrm{C}}$ and KRAS ${ }^{\mathrm{Q} 61 \mathrm{H}}$ protein with a moderate binding affinity of $-5.38,-5.41$, and -3.97 kcal/mol, respectively. In addition, 0375-0604 selectively inhibited the proliferation of NSCLC cells with KRAS mutation but not normal lung cells. Compound 0375-0604 also blocked the formation of GTP-KRAS and downstream activation of KRAS in vivo. Besides, compound 0375-0604 inhibited the growth of cancer cells by causing G2/M cell cycle arrest and inducing apoptosis. Regardless, our study provides further evidence for targeting to KRAS protein, which may contribute to the future study for lung cancer therapy.

\section{MATERIALS AND METHODS}

\section{Molecular Docking}

The KRAS ${ }^{G 12 D}$ structure (PDB code: 4DSU) complexed with GDP and a compound benzimidazole (BZIM) was used for the modeling of possible binding modes between KRAS and 03750604. The crystal structure with GDP was prepared in the Prep Wiz module of Maestro (Version 9.1, Schrodinger) and water molecules within $5 \AA$ of het groups were kept. Subsequently, the residues of D12 and Q61 were mutated into C12 and H61 using the BioLuminate module of Maestro, respectively. A grid file was generated based on the position of compound BZIM in the Grid Generation wizard. Then, 0375-0604 was prepared to assign atomic charges and generate alternative conformations chemical rings. Finally, the docking process was employed in the Glide Docking module based on the previous obtained grid file using an extra precision (XP) protocol followed by a post-docking minimization using MM-GBSA method.

\section{Biotinylation}

KRAS (Abcam, ab96817; $200 \mu \mathrm{g} / \mathrm{ml}$ ) was biotinylated using the EZ-Link NHS-LC-LC-biotin (Thermo) in $\mathrm{H}_{2} \mathrm{O}$ using a 3:1 molar ratio of biotin reagent: protein for $30 \mathrm{~min}$ at room temperature following the FortéBio suggested protocol. Biotinylated KRAS was separated from the biotinylation reaction reagents by Zeba desalying spin columns (Thermo). Streptavidin biosensors (SA) tips (Forteìio, Inc., Menlo Park, CA, United States) were prewetted with PBS to establish a baseline before immobilization.

\section{Biolayer Interferometry Analysis}

A FortéBio Octet Red instrument was used in this assay. All the assays were performed at 96-well plate (Greiner BioOne, PN:655209) and all the final volume for all the solutions was $200 \mu \mathrm{l} /$ well. Biotinylated KRAS was immobilized onto SA tips. The experiments comprised three steps: (1) baseline, (2) association, (3) dissociation. The association and dissociation plot and kinetic constants were obtained with Forteìio data analysis software. For measurement the interaction between 
compound 0375-0604 and KRAS, seven concentrations of compound 0375-0604 (20, 40, 60, 80, 100, 120, 140, $160 \mu \mathrm{M})$ were used for association step.

\section{3-(4, 5-Dimethylthiazol-2-yl)-2, 5-Diphenyltetrazolium Bromide (MTT) Assay}

H2122, H460 and H358 were purchased from ATCC and cultivated with RPMI 1640 medium supplemented with $10 \%$ fetal bovine serum (FBS), 100 units $/ \mathrm{mL}$ penicillin and $100 \mu \mathrm{g} / \mathrm{mL}$ streptomycin. All the cells were cultivated at $37^{\circ} \mathrm{C}$ with $5 \%$ $\mathrm{CO}_{2}$ incubator. Cells were seeded on a 96-well microplate with 3000,4000 , or 5000 cells/well, and were cultured overnight for cell adhesion. After add a range of compound 0375-0604 the microplates put back into incubator and incubated for 24,48 , or $72 \mathrm{~h}$. Each dosage was repeated as triplicate. $10 \mu \mathrm{L}$ MTT $(5 \mathrm{mg} / \mathrm{mL})$ solutions were added to each well. After incubated $4 \mathrm{~h} 100 \mu \mathrm{L}$ DMSO was added to each well. After 15 min shake absorbance of the plate was measured at $570 \mathrm{~nm}$ (absorbance) and $650 \mathrm{~nm}$ (reference) by a microplate reader (Tecan).

\section{Pull Down Assay}

RAS activity was determined using RAS activation assay kit (EMD Millipore, 17-218). Briefly, lysates were incubated with glutathione S-transferase fusion of the Ras binding domain (RBD) of Raf1 along with glutathione agarose for $1 \mathrm{~h}$. Agarose beads were collected by centrifugation and washed three times with $\mathrm{Mg}^{2+}$ lysis buffer. Each sample were resuspended and boiled for $5 \mathrm{~min}$. Finally, samples were subjected to western blotting as previously described and blots probed using anti-KRAS antibody (Santa, sc-30).

\section{Western Blot Analysis}

After $48 \mathrm{~h}$ treatment with compound 0375-0604, RIPA lysis buffer (150 mM NaCl, $50 \mathrm{mM}$ Tris $\mathrm{pH}$ 7.4, 1 mM EDTA, $0.25 \%$ sodium deoxycholate, $1 \%$ NP-40) containing protease (Roche) and phosphatase (Roche) inhibitors was added to extract the total whole cell protein. Bio-Rad $\mathrm{DC}^{\mathrm{TM}}$ protein assay kit was used to quantify the concentration of extract protein. Thirty microgram protein lysate was loaded and separated by $10 \%$ SDS-polyacrylamide gel electrophoresis and transferred to a nitrocellulose (Millipore) membrane. The membrane was incubated with the primary antibody (1:2000) and then with a

\section{REFERENCES}

Athuluri-Divakar, S. K., Vasquez-Del Carpio, R., Dutta, K., Baker, S. J., Cosenza, S. C., Basu, I., et al. (2016). A small molecule RAS-mimetic disrupts RAS association with effector proteins to block signaling. Cell 165, 643-655. doi: 10.1016/j.cell.2016.03.045

Berndt, N., Hamilton, A. D., and Sebti, S. M. (2011). Targeting protein prenylation for cancer therapy. Nat. Rev. Cancer 11, 775-791. doi: 10.1038/nrc3151

Bos, J. L., Rehmann, H., and Wittinghofer, A. (2007). GEFs and GAPs: critical elements in the control of small G proteins. Cell 129, 865-877. doi: 10.1016/j. cell.2007.05.018

Brock, E. J., Ji, K., Reiners, J. J., and Mattingly, R. R. (2016). How to target activated RAS proteins: direct inhibition $v s$. induced Mislocalization. fluorescence-conjugated secondary antibody (1:10000). GAPDH was used as the loading control and for normalization. The signal of the membranes was scanned with a LI-COR Odyssey Scanner (Belfast).

\section{Cell Cycle and Apoptosis Assay Using Flow Cytometry}

H2122, H358 and H460 cells were plated on a 6-well plate with $1.5 \times 10^{5}$ cells/well and cultured overnight for attachment. After treatment with compound 0375-0604 at 0, 25, 50, $100 \mu \mathrm{M}$ for 24, 48, and $72 \mathrm{~h}$, all cells were harvested and collected. For cell cycle analysis, cells pellets were re-suspended in 70\% ethanol and fixation at $4^{\circ} \mathrm{C}$ for $30 \mathrm{~min}$. Each cell pellet was stained in $300 \mu \mathrm{L}$ propodium iodide (PI) $(50 \mu \mathrm{g} / \mathrm{ml})$ staining solution at $37^{\circ} \mathrm{C}$ for $30 \mathrm{~min}$ in dark. Then, cells were washed twice with PBS. Finally, cells were re-suspended in $300 \mu \mathrm{L}$ PBS and transferred to the flow cytometer (FACSCalibur, BD Biosciences). For apoptosis analysis, cells will re-suspended with $100 \mu \mathrm{L}$ annexin-binding buffer, stained with annexin $\mathrm{V}$ and PI staining solution and incubated $15 \mathrm{~min}$ at room temperature protect from light. Finally, cells were diluted in $300 \mu \mathrm{L}$ annexin-binding buffer and quantitatively measured using flow cytometer (FACSCalibur, BD Biosciences).

\section{Statistical Analysis}

Descriptive analytical data are presented as means \pm SD. Statistical analysis was conducted using Graph Prim 5.0. Significant differences between datasets were assessed by one-way analysis of variance (ANOVA).

\section{AUTHOR CONTRIBUTIONS}

X-JY, EL-HL, and LL conceived the project. X-JY, EL-HL, and CX designed the experiments. CX, YL, L-LL, X-XF, Y-WW, and C-LW carried out the research and analysis of data. X-JY, EL-HL, $\mathrm{LL}$, and CX wrote the paper.

\section{ACKNOWLEDGMENT}

This work was supported by Macao Science and Technology Development Fund (Project No: 082/2013/A3 \& 086/2015/A3 \& 005/2014/AMJ\&046/2016/A2).

Mini. Rev. Med. Chem. 16, 358-369. doi: 10.2174/13895575156661510011 54002

Bryant, K. L., Mancias, J. D., Kimmelman, A. C., and Der, C. J. (2014). KRAS: feeding pancreatic cancer proliferation. Trends Biochem. Sci. 39, 91-100. doi: 10.1016/j.tibs.2013.12.004

Burns, M. C., Sun, Q., Daniels, R. N., Camper, D., Kennedy, J. P., Phan, J., et al. (2014). Approach for targeting Ras with small molecules that activate SOSmediated nucleotide exchange. Proc. Natl. Acad. Sci. U.S.A. 111, 3401-3406. doi: 10.1073/pnas.1315798111

Chavan, T. S., Muratcioglu, S., Marszalek, R., Jang, H., Keskin, O., Gursoy, A., et al. (2015). Plasma membrane regulates Ras signaling networks. Cell. Logist. 5:e1136374. doi: 10.1080/21592799.2015.11 36374 
Chen, Z., Fillmore, C. M., Hammerman, P. S., Kim, C. F., and Wong, K. K. (2014). Non-small-cell lung cancers: a heterogeneous set of diseases. Nat. Rev. Cancer 14, 535-546. doi: 10.1038/nrc3775

Clausen, R., Ma, B., Nussinov, R., and Shehu, A. (2015). Mapping the conformation space of wildtype and mutant $\mathrm{H}$-Ras with a memetic, cellular, and multiscale evolutionary algorithm. PLOS Comput. Biol. 11:e1004470. doi: 10.1371/journal. pcbi. 1004470

Cox, A. D., Der, C. J., and Philips, M. R. (2015). Targeting RAS membrane association: back to the future for anti-RAS drug discovery? Clin. Cancer Res. 21, 1819-1827. doi: 10.1158/1078-0432.CCR-14-3214

Cromm, P. M., Spiegel, J., Grossmann, T. N., and Waldmann, H. (2015). Direct modulation of small GTPase activity and function. Angew. Chem. Int. Ed. Engl. 54, 13516-13537. doi: 10.1002/anie.201504357

Downward, J. (2003). Targeting RAS signalling pathways in cancer therapy. Nat. Rev. Cancer 3, 11-22. doi: 10.1038/nrc969

Ettinger, D. S., Wood, D. E., Aisner, D. L., Akerley, W., Bauman, J., Chirieac, L. R., et al. (2017). Non-small cell lung cancer, version 5.2017, NCCN clinical practice guidelines in oncology. J. Natl. Compr. Canc. Netw. 15, 504-535. doi: 10.6004/jnccn.2017.0050

Gysin, S., Salt, M., Young, A., and Mccormick, F. (2011). Therapeutic strategies for targeting ras proteins. Genes Cancer 2, 359-372. doi: 10.1177/ 1947601911412376

Jancik, S., Drabek, J., Radzioch, D., and Hajduch, M. (2010). Clinical relevance of KRAS in human cancers. J. Biomed. Biotechnol. 2010:150960. doi: 10.1155/2010/ 150960

Karachaliou, N., Mayo, C., Costa, C., Magrí, I., Gimenez-Capitan, A., Molina-Vila, M. A., et al. (2013). KRAS mutations in lung cancer. Clin. Lung Cancer 14, 205. doi: $10.1016 /$ j.cllc.2012.09.007

Keeton, A. B., Salter, E. A., and Piazza, G. A. (2017). The RAS-effector interaction as a drug target. Cancer Res. 77, 221-226. doi: 10.1158/0008-5472.CAN-16-0938

Laheru, D., Shah, P., Rajeshkumar, N. V., Mcallister, F., Taylor, G., Goldsweig, H., et al. (2012). Integrated preclinical and clinical development of S-trans, transfarnesylthiosalicylic Acid (FTS, Salirasib) in pancreatic cancer. Invest. New Drugs 30, 2391-2399. doi: 10.1007/s10637-012-9818-6

Lazo, J. S., and Sharlow, E. R. (2016). Drugging undruggable molecular cancer targets. Annu. Rev. Pharmacol. Toxicol. 56, 23-40. doi: 10.1146/annurevpharmtox-010715-103440

Ledford, H. (2015). Cancer: the Ras renaissance. Nature 520, 278-280. doi: 10.1038/ $520278 \mathrm{a}$

Leshchiner, E. S., Parkhitko, A., Bird, G. H., Luccarelli, J., Bellairs, J. A., Escudero, S., et al. (2015). Direct inhibition of oncogenic KRAS by hydrocarbon-stapled SOS1 helices. Proc. Natl. Acad. Sci. U.S.A. 112, 1761-1766. doi: 10.1073/pnas. 1413185112

Lito, P., Solomon, M., Li, L.-S., Hansen, R., and Rosen, N. (2016). Allele-specific inhibitors inactivate mutant KRAS G12C by a trapping mechanism. Science 351, 604-608. doi: 10.1126/science.aad6204

Lu, S. Y., Jang, H., Gu, S., Zhang, J., and Nussinov, R. (2016). Drugging Ras GTPase: a comprehensive mechanistic and signaling structural view. Chem. Soc. Rev. 45, 4929-4952. doi: 10.1039/c5cs00911a

Maurer, T., Garrenton, L. S., Oh, A., Pitts, K., Anderson, D. J., Skelton, N. J., et al. (2012). Small-molecule ligands bind to a distinct pocket in Ras and inhibit SOS-mediated nucleotide exchange activity. Proc. Natl. Acad. Sci. U.S.A. 109, 5299-5304. doi: 10.1073/pnas.1116510109

Papke, B., and Der, C. J. (2017). Drugging RAS: know the enemy. Science 355, 1158-1163. doi: 10.1126/science.aam7622

Prakash, P., and Gorfe, A. A. (2013). Lessons from computer simulations of Ras proteins in solution and in membrane. Biochim. Biophys. Acta 1830, 5211-5218. doi: 10.1016/j.bbagen.2013.07.024
Pylayeva-Gupta, Y., Grabocka, E., and Bar-Sagi, D. (2011). RAS oncogenes: weaving a tumorigenic web. Nat. Rev. Cancer 11, 761-774. doi: 10.1038/nrc3106

Rich, R. L., and Myszka, D. G. (2007). Higher-throughput, label-free, real-time molecular interaction analysis. Anal. Biochem. 361, 1-6. doi: 10.1016/j.ab.2006. 10.040

Rowinsky, E. K., Windle, J. J., and Von Hoff, D. D. (1999). Ras protein farnesyltransferase: a strategic target for anticancer therapeutic development. J. Clin. Oncol. 17, 3631-3652. doi: 10.1200/JCO.1999.17.11.3631

Santarpia, L., Lippman, S. M., and El-Naggar, A. K. (2012). Targeting the MAPKRAS-RAF signaling pathway in cancer therapy. Expert Opin. Ther. Targets 16, 103-119. doi: 10.1517/14728222.2011.645805

Shima, F., Matsumoto, S., Yoshikawa, Y., Kawamura, T., Isa, M., and Kataoka, T. (2015). Current status of the development of Ras inhibitors. J. Biochem. 158, 91-99. doi: 10.1093/jb/mvv060

Smith, M. J., Neel, B. G., and Ikura, M. (2013). NMR-based functional profiling of RASopathies and oncogenic RAS mutations. Proc. Natl. Acad. Sci. U.S.A. 110, 4574-4579. doi: 10.1073/pnas. 1218173110

Trinh, T. B., Upadhyaya, P., Qian, Z., and Pei, D. (2016). Discovery of a direct Ras inhibitor by screening a combinatorial library of cell-permeable bicyclic peptides. ACS Comb. Sci. 18, 75-85. doi: 10.1021/acscombsci.5b00164

Upadhyaya, P., Bedewy, W., and Pei, D. (2016). Direct inhibitors of Raseffector protein interactions. Mini Rev. Med. Chem. 16, 376-382. doi: 10.2174/ 1389557515666151001141713

Van Cutsem, E., Van De Velde, H., Karasek, P., Oettle, H., Vervenne, W. L., Szawlowski, A., et al. (2004). Phase III trial of gemcitabine plus tipifarnib compared with gemcitabine plus placebo in advanced pancreatic cancer. J. Clin. Oncol. 22, 1430-1438. doi: 10.1200/JCO.2004.10.112

Vasan, N., Boyer, J. L., and Herbst, R. S. (2014). A RAS renaissance: emerging targeted therapies for KRAS-mutated non-small cell lung cancer. Clin. Cancer Res. 20, 3921-3930. doi: 10.1158/1078-0432.ccr-13-1762

Vo, U., Vajpai, N., Flavell, L., Bobby, R., Breeze, A. L., Embrey, K. J., et al. (2016). Monitoring Ras interactions with the nucleotide exchange factor son of Sevenless (Sos) using site-specific NMR reporter signals and intrinsic fluorescence. J. Biol. Chem. 291, 1703-1718. doi: 10.1074/jbc.M115. 691238

Wang, Q., Qi, Y., Yin, N., and Lai, L. (2014). Discovery of novel allosteric effectors based on the predicted allosteric sites for Escherichia coli D-3-phosphoglycerate dehydrogenase. PLOS ONE 9:e94829. doi: 10.1371/journal.pone.009 4829

Wang, W., Fang, G., and Rudolph, J. (2012). Ras inhibition via direct Ras bindingis there a path forward? Bioorg. Med. Chem. Lett. 22, 5766-5776. doi: 10.1016/j. bmcl.2012.07.082

Zimmermann, G., Papke, B., Ismail, S., Vartak, N., Chandra, A., Hoffmann, M., et al. (2013). Small molecule inhibition of the KRAS-PDEdelta interaction impairs oncogenic KRAS signalling. Nature 497, 638-642. doi: 10.1038/ nature 12205

Conflict of Interest Statement: The authors declare that the research was conducted in the absence of any commercial or financial relationships that could be construed as a potential conflict of interest.

Copyright (c) 2017 Xie, Li, Li, Fan, Wang, Wei, Liu, Leung and Yao. This is an open-access article distributed under the terms of the Creative Commons Attribution License (CC BY). The use, distribution or reproduction in other forums is permitted, provided the original author(s) or licensor are credited and that the original publication in this journal is cited, in accordance with accepted academic practice. No use, distribution or reproduction is permitted which does not comply with these terms. 